\title{
Applying neural network for the segmentation of spike structural elements
}

\author{
Epifanov R.1,3*, Genaev M.1,2,3 \\ ${ }^{1}$ Novosibirsk State University, Novosibirsk, Russia \\ ${ }^{2}$ Institute of Cytology and Genetics, SB RAS, Novosibirsk, Russia \\ ${ }^{3}$ Kurchatov Genomic Center of the Institute of Cytology and Genetics, SB RAS, Novosibirsk, Russia \\ * email: rostepifanov@gmail.com
}

The shape and structure of the wheat spike is one of the most important characteristics of cereals associated with their economically valuable qualities such as productivity, the absence of ear fragility and ease of threshing. The study of the genes controlling these traits will allow us to purposefully create new varieties with improved characteristics in terms of yield, ease of thresh and resistance to environmental factors. Evaluation of wheat spike characteristics in most modern studies is performed by an expert based on the visual analysis and measuring practices, which requires a significant investment of time, despite the fact that in modern experiments tens of thousands of plants are analyzed. Automation of this time-consuming process through the introduction of digital image analysis technologies is relevant for modern science. Important step in wheat morphometric analysis is spike structural element segmentation at body and awns of spike. In this work, we propose a method based on neural networks to segment ear structural elements. The algorithm is based on usage of Unet. Model accuracy according to the IoU metric was $94.73 \%$ for the body and $77.70 \%$ for the awns of wheat, which is 2.23 and $11.70 \%$ higher than that of the WERecognizer model posted on wheatdb.org/werecognizer.

Acknowledgements: This work was supported by the Kurchatov Genomic Center of the Institute of Cytology and Genetics, SB RAS (agreement No. 075-15-2019-1662). 